\title{
An Improved Multimodal Palm Vein Recognition System Using Shape and Texture Features
}

\author{
M.Deepamalar and M.Madheswaran
}

\begin{abstract}
Multimodal analysis of palm vein for biometric authentication has been carried out and presented in this paper. The proposed system is based on parallel mode multiple feature analysis and multilevel fusion. The directional information of the palm vein has been considered for better analysis. The developed multimodal palm vein recognition system has very low value of matching error rate and false acceptance or rejection rate.
\end{abstract}

Index Terms-Palm vein recognition, Multimodal Biometrics, Feature subset selection, ASFFS, FAR, FRR

\section{INTRODUCTION}

The advancement in biometric image matching technology has promoted the development of various authentication systems. Hitachi and Fujitsu [1] have fabricated and introduced finger vein authentication system in 2004 and 2006 respectively. The palm vein authentication system is introduced by Fujitsu, uses vascular patterns of palm and finger for efficient and robust authentication. Mohamed Shahin et al [2] demonstrated the hand vein pattern authentication technology. A personal verification system using the thermal-imaged vein pattern in the back of the hand is proposed by Lingyu Wang and Graham Leeham [3]. Jian-Gang Wang et al. [4] proposed a multimodal personal identification system, in which palmprint and palm vein images are fused by a novel integrated line-preserving and contrast-enhancing method. Zhongli Wang et al. [5] demonstrated the performance evaluation of shape and texture based methods for vein recognition. Chih-Lung Lin and Kuo-Chin Fan [6] presented a novel approach to personal verification using the thermal images of palm-dosra vein patterns. Multiresolution representation of images with feature points of the vein patterns (FPVP) are obtained using multiple resolution filters that extract the dominant points by filtering miscellaneous features for each FPVP.

A prototype low cost automatic thermo graphic imaging system which has been developed by J.M.Cross and C.L.Smith [7] to obtain vein patterns for positive identification and describe to use the subcutaneous vascular network of the back of the hand as a unique personal biometric for identification. Vein image processing includes threshold segmentation and thinning of the vein pattern are demonstrated by Y.Ding et al [8].

M.Wantanbe et al [9] provides software for vein pattern

\footnotetext{
${ }^{1}$ M.Deepamalar and M.Madheswaran are with Centre for Advanced Research, Electronics and Communication Engineering, Muthayammal Engineering College, Rasipuram - 637 408, India. Phone:91-4287-226837; e-mail: madheswaran.dr@gmail.com
}

authentication. This vein pattern authentication software translates the black lines into a blood vessel pattern of the palm, and then matches the translated vein pattern with a pre-registered template pattern, while correction for position and orientation of the palm by a pattern matching method.

The techniques presented for personal authentication $[3$, 5-9] are based on unimodal biometric system, which have some limitations such as noise in sensed data, intra-class variations, distinctiveness, and nonuniversality and spoof attacks. The "Laplacian" and Junction Point approach multimodal presented in $[4,10]$ are based on fusion at imaging level. One of the major issues in imaging fusion is image alignment or registration, which refer to pixel-bypixel alignment of the images. The proposed palm vein recognition system is expected to overcome some of the limitations of the existing work.

\section{Multmodal Palm Vein Recognition System}

Automatic recognition of palm vein pattern can be focused on two tasks, learning the invariant and recognition of palm vein patterns. The task of palm vein pattern recognition by a computer can be described as a transformation from the measurement space $\mathrm{M}$ to the decision space D. The mapping function $M \rightarrow D$ is described by the extended transition function of $\bar{\delta}\left(s_{0}, M\right) \Rightarrow s_{4}^{f}$. The elements of $\mathrm{M}$ are preprocessed palm vein image $M=\left\{m_{1}, m_{2}, \ldots \ldots, m_{n}\right\}, m_{i} \in M$, the elements of $F$ are multiple features set $F=\left\{f_{1}, f_{2}, \ldots \ldots, f_{n}\right\}, f_{i} \in F$, the elements of $\mathrm{O}$ are optimized feature set $O=\left\{o_{1}, o_{2}, \ldots \ldots, o_{n}\right\}, o_{i} \in O$ and the elements $\mathrm{D}$ are termed as decision $D=\left\{d_{1}, d_{2}, \ldots \ldots, d_{n}\right\}, d_{i} \in D$

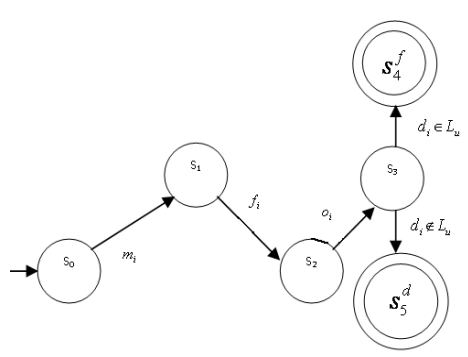

Fig 1. Multimodal Palm Vein Recognition System

A multimodal palm vein recognition system can be 
constructed

using

five

states $S=\left\{S_{0}, S_{1}, S_{2}, S_{3}, S_{4}^{f}, S_{5}^{d}\right\}$, which can be defined by the quintuple $R=\left(S, \sum, S_{0}, \delta, F\right)$, where $\mathrm{S}$ is the total number of states in the palm vein recognition system. $S_{0}$ represents the start or initial state, which can perform palm vein image acquisition and preprocessing to provide the measurement space $\mathrm{M}, S_{1}$ represents the multiple feature extraction state to provide the multiple feature set F, $S_{2}$ denotes the feature optimization state to provide the optimal feature space $\mathrm{O}$ and $S_{3}$ refers the decision state to give decision space D. The two states $S_{4}^{f}, S_{5}^{d}$ define final state to accept the legitimate user and dead state to reject the imposter respectively. In the quintuples $\sum$ the optimal multiple feature vector, which is called as input patterns. The transition map can be denoted as $\delta: S_{0} \times \sum \rightarrow S$ and defines the recognition process from the measurement space to the decision space.

The extended transition function represents the recognition process from the initial state to final state is denoted by $\bar{\delta}\left(S_{0}, W d_{i}\right) \rightarrow s_{4}^{f}$. This can be expanded as follows:

$$
\begin{gathered}
\delta\left(\bar{\delta}\left(S_{0}, W O_{i}\right), d_{i}\right) \rightarrow S_{4}^{f} \\
\delta\left(\delta\left(\bar{\delta}\left(S_{0}, W f_{i}\right), o_{i}\right), d_{i}\right) \rightarrow S_{4}^{f} \\
\delta\left(\delta\left(\delta\left(\bar{\delta}\left(S_{0}, m_{i}\right), f_{i}\right), o_{i}\right), d_{i}\right) \rightarrow S_{4}^{f}
\end{gathered}
$$

This transition function can be represented by transition table as follows:

\begin{tabular}{|c|c|c|c|c|c|}
\hline \multirow{2}{*}{ States } & \multicolumn{5}{|c|}{ Input Symbols } \\
\cline { 2 - 6 } & $m_{i}$ & $f_{i}$ & $o_{i}$ & $d_{i} \in L_{u}$ & $d_{i} \notin L_{u}$ \\
\hline$S_{0}$ & $S_{1}$ & -- & -- & -- & -- \\
\hline$S_{1}$ & -- & $S_{2}$ & -- & -- & -- \\
\hline$S_{2}$ & -- & -- & $S_{3}$ & -- & -- \\
\hline$S_{3}$ & -- & -- & -- & $S_{4}^{f}$ & $S_{5}^{d}$ \\
\hline
\end{tabular}

In multimodal biometric system, multiple units of the same biometrics are combined. Depending on the number of traits, sensors and feature sets used in multimodal biometric system information reconciliation can be done by any of the fusion type, fusion at feature extraction level, fusion at matching score level and fusion at decision level [22]. The proposed multimodal recognition system is based on the convolved shape and texture features in a multiple feature set, skeleton, bifurcation and ending points of the palm vein image can be considered. Multimodal is designed by parallel mode fusion on biometric units at different levels such as feature extraction level and decision level. And also this proposed system can construct the matching module using two different classifiers such as k-NN (k-Nearest Neighbor) and ART1 (Adaptive Resonance Theory). The performance of $\mathrm{k}-\mathrm{NN}$ and ART1 on multimodal palm vein recognition system can be compared in the proposed system.
The multimodal palm vein recognition system can be constructed by five phases such as preprocessing module, feature Extraction module, feature optimization and subset selection, vein Pattern Matching module and decision module.

\section{A. Preprocessing}

The vein image is in poor contrast due to glare, and contains irregular shading caused by various thicknesses of skin and bones. Vein pattern authentication requires a normalized and enhanced vein image to authenticate a reliable user. Near Infra Red Vein imaging techniques are used to capture the palm vein image [4]. Guy Gilboa, Nir Sochen, and Yehoshua Y.Zeevi introduce complex Shock filters. This paper presents a de-noising and enhancement technique based on GSZ - shock filter, which focuses on both noise elimination and edge enhancement. This approach is an edge preserving technique based on nonlinear diffusion, in which the image gradient has been used to weight the diffusion process in order to smooth the vein image while preserving the vein edge. They derive the complex shock filter formulation for small $\theta$ [11-13].

\section{B. Feature Extraction At Various Levels}

In this proposed system Multiple Feature extraction technique extracts hand shape features, which is convolved with texture feature to get the multiple feature set, this process is called as fusion at feature extraction level. This phase also extracts skeleton, bifurcation and ending points of the palm vein image.

\section{i. Fusion at extraction level}

Fusion at feature extraction level is responsible to provide geometrical feature to texture of the palm vein image. Normally the texture is not consisting of any geometrical features, so the shape feature can be convolved with the palm texture to add geometrical features, which can make the convolved feature set as a higher priority feature set for recognition. The picture of palm is shown in fig 3 . Shape feature of the palm is extracted by the palm size $\|\mathrm{A} 1 \mathrm{~A} 2\|$ and the distance between $\mathrm{O} 1$ and $\mathrm{O} 2$. The values $\mathrm{O} 1$ and $\mathrm{O} 2$ are computed by calculating the Euclidean distance between the points X [L1] and X [L2]. Where L1 and L2 are fixed indexes obtained by trial and error basis.

$$
\begin{aligned}
& \|O 1 O 2\|=\alpha\|X[L 1] X[L 2]\| \\
& \|A 1 A 2\|=\beta\|X[L 1] X[L 2]\|
\end{aligned}
$$

where $\alpha$ and $\beta$ are the dimensions coefficient. Then the palm size is resized with the fixed size $M^{*} \mathrm{M}$, where $\mathrm{M}$ is as 100 .

The texture features are obtained using Gabor wavelet. Normally the Gabor features provide the visual properties such as the spatial location, orientation selectivity, and spatial frequency characteristics. The Gabor wavelets can be given by [5] as follows

$$
\begin{aligned}
& G_{u, v}(z)=\left(\left\|k_{u, v}\right\|^{2} / \sigma^{2}\right) e^{\left(-\left\|k_{u, v}\right\|^{2}\|z\|^{2} / 2 \sigma^{2}\right)}\left[e^{i k_{u, v}{ }^{*} z}-e^{-\sigma^{2} / 2}\right] \\
& k_{u, v}=\left(\begin{array}{l}
k_{v} \cos \Phi_{u} \\
k_{v} \sin \Phi_{u}
\end{array}\right), \quad k_{v}=\frac{f_{\max }}{2^{v+2}}, \quad \Phi_{u}=u \frac{\pi}{8}, \quad v
\end{aligned}
$$


where $v$ is the scale of the kernal and $u$ is the orientation with a spatial frequency $f$ fixed at $1.26, \Phi$ controls the function orientation, $\sigma$ is the standard deviation of the Gaussian factor and $\mathrm{z}=(\mathrm{x}, \mathrm{y})^{\mathrm{T}}$. The Gabor wavelets can be used to enhance the features in certain scales and orientations.

Let $\mathrm{Gu}, \mathrm{v}(\mathrm{Z})$ denotes the Gabor magnitude feature of an image, where $\mathrm{u}$ and $\mathrm{v}$ are the orientation and scale of the kernel (k), respectively. The feature extraction is based on the multi-region mean and Gabor magnitude feature and can be represented as [5]

$$
\overline{G \mu_{u, v, l}}=\frac{1}{N} \sum_{Z_{i}=R_{l}} G_{u, v}\left(Z_{j}\right)
$$

where $1=1,2, \ldots \mathrm{L}$ is the region index, $\mathrm{L}$ is the number of sub-region and $\mathrm{N}$ represents the total number of pixels in the region $\mathrm{Rl}$. The palm features can be obtained by convolving palm vein input image with the Gabor filter by [15]

$$
c(x, y)=I(x, y) \otimes G_{u, v}(z)
$$

The distance between the dominant points obtained by using (4) \& (5) can be used to approximately calculate the extracted hand shape characteristics such as widths and lengths.

$$
\begin{aligned}
& L[i]=d\left(m\left(X\left[H_{2 i}\right], X\left[H_{2 i+2}\right]\right), X\left[H_{2 i+l}\right]\right) \\
& \left.l[i][j]=d\left(X\left\lfloor H_{2 i+1-j}\right\rfloor, X \mid H_{21+1+j}\right\rfloor\right)
\end{aligned}
$$

where $d(a, b)$ is the Euclidean distance between the points a and $b, m(a, b)$ returns the medium of the segment $\|a b\|, L[i]$ is the $\mathrm{i}^{\text {th }}$ finger length, $l[\mathrm{i}][\mathrm{j}]$ is the $\mathrm{j}^{\text {th }}$ width of the $\mathrm{i}^{\text {th }}$ finger and $\mathrm{Hi}=\mathrm{i}(\mathrm{N}+l)$ is the index of the $\mathrm{i}^{\text {th }}$ initial characteristics point of the hand, which are shown in fig 2 .

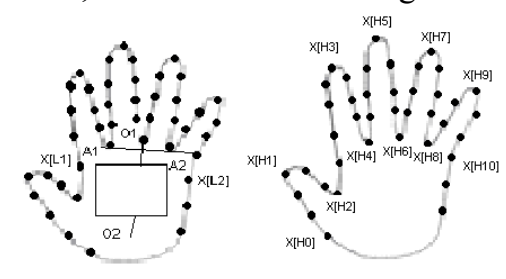

Fig 2. Hand Shape Features

The fusion of palm vein texture obtained by Gabor filters and hand shape features can be achieved using convolution. The result of the convolution is binarized and the process is defined by[15]

$$
F(x, y)=b(C(x, y) \otimes H(x, y))
$$

where $\mathrm{b}(\mathrm{x})=0$ if $\mathrm{x}<0$ and $\mathrm{b}(\mathrm{x})=1$ otherwise. $\mathrm{H}$ is the $5 \mathrm{X} 5$ filter based on the shape feature. $\mathrm{H}$ filter is defined by:

$$
H(x, y)=H^{\prime}(x, y) \times \frac{1}{\sum_{i=0}^{4} \sum_{j=0}^{4} H^{\prime}[i][j]}
$$

where $H^{\prime}$ (The normalization coefficient) =

\begin{tabular}{|l|l|l|l|l|}
\hline $\mathrm{L}[0]$ & $l[0][1]$ & $l[0][2]$ & $l[0][3]$ & $l[0][4]$ \\
\hline $\mathrm{L}[1]$ & $l[1][1]$ & $l[1][2]$ & $l[1][3]$ & $l[1][4]$ \\
\hline $\mathrm{L}[2]$ & $l[2][1]$ & $l[2][2]$ & $l[2][3]$ & $l[2][4]$ \\
\hline $\mathrm{L}[3]$ & $l[3][1]$ & $l[3][2]$ & $l[3][3]$ & $l[3][4]$ \\
\hline $\mathrm{L}[4]$ & $l[4][1]$ & $l[4][2]$ & $l[4][3]$ & $l[4][4]$ \\
\hline
\end{tabular}

\section{ii. Skeleton Extraction}

A palm vein pattern can be well represented by its skeleton. The skeleton of the vein pattern is extracted by the skeletonization algorithm based on local threshold and local mean value [5]. The directional information is obtained by including eight steps in skeletonization process, where each step peels one layer of pixels in a certain direction. After each iteration is accomplished, the pattern is one pixel thinner in all directions. When there is no difference between the input and output the algorithm will be terminated [15]. Different skeleton templates are applied to the corresponding direction of "Peeling". The feedback templates A are set to zero and the bias valued is set to -3 for every step. The skeleton templates used in the proposed method have been introduced by S. Malki et al [15].

\section{iii. Bifurcation and Ending points extraction}

Palm vein patterns contained two main features, ending and bifurcation. The endings are the end points of a thinned line and the bifurcation is the junction point of three lines. If the current pixel in the skeleton feature is $(\mathrm{xc}, \mathrm{yc})$ and then its neighboring pixels can defined as $\mathrm{Nr}(\mathrm{xc}, \mathrm{yc})$ in a $3 \times 3$ matrix.

$$
N_{c}=T_{c} \cap N_{r}\left(x_{c}, y_{c}\right)
$$

where $\mathrm{Nc}$ is the number of neighboring pixels of (xc,yc). Tc is the pixel set available in skeleton feature.

$$
N_{c}=T_{c} \cap N_{r}\left(x_{c}, y_{c}\right)= \begin{cases}\left(x_{c}, y_{c}\right) \in E_{f}, & N_{c}=1 \\ \left(x_{c}, y_{c}\right) \in V_{f}, & N_{c}=2 \\ \left(x_{c}, y_{c}\right) \in B_{f}, & N_{c}=3 \\ \left(x_{c}, y_{c}\right) \in \text { false-bifurcation, }, & N_{c}>3\end{cases}
$$

where $\mathrm{Bf}$ is a set of bifurcations, Ef is a set of ending points and $\mathrm{Vf}$ is a set of pixel in the vein path.

\section{Feature Optimization}

The feature selection or optimization is responsible to select a hitherto slighter subset of the extracted features that are estimated to be the most distinctive and instructive ones. Adaptive sequential floating forward search (ASFFS) is one of the best feature selection methods. The ASFFS algorithm described by Somol.P et al is considered to optimize the palm vein features. Most significant feature can be added with current subset and the least significant feature can be conditionally removed from the current subset iteratively by this method. ASFFS starts from the empty set. After each forward adding step, it performs backward conditional removing steps as long as the objective function $(\mathrm{Az})$ of the current subset increases [16].

The feature subset selection using ASFFS considering of two phases such as forward phase and backward phase with the initial set up as the best values of decisive factor function $J\left(X_{i}\right)$ are stored as $J_{i}^{\max }$ for all i. The consequent so - far best feature subsets $\mathrm{Xi}$ are also stored. Initial values of $J_{i}^{\max }$ for all $\mathrm{i}$ should be set to lowest position value. $\mathrm{k}$ is the size of the current subset. The algorithm is initialized by setting $\mathrm{k}=0$ and $\mathrm{X} 0=0$.

\section{i. ASFFS Algorithm}

Each phase begins with adaptive setting of $\mathrm{r}$ :

$$
\text { If }|k-d|<b \text {, let } r=r_{\text {max }} \text {, }
$$


Else if $|k-d|<r_{\max }+b$, let $r=r_{\max }+b-|k-d|$

Else let $r=1$ where $\mathrm{d}$ is the palm vein feature subset fining from the $\mathrm{D}$ measurements, $\mathrm{d}<\mathrm{D}$ has been the interest for long time, $\mathrm{k}$ is the current subset size, $r_{\max }$ is a user specified absolute generalization limit, $r$ is the actual generalization limit for the current subset $\left(\mathrm{r}<r_{\max }\right), \mathrm{b}$ is the neighborhood of the final dimension and o is the current generalization level depending on the current situation $(o \leq r)$.The flow graph of the ASFFS algorithm is shown in fig 7 .

\section{Forward Phase steps:}

1. Initialize $\mathrm{o}=1$

2. Perform condition inclusion with respect to the feature set.

3. Find out the so-far best subset of size $\mathrm{k}+\mathrm{o}$

4. Perform conditional increase of generalization step. The conditionally included features are removed.

5. None of the subset tested in the forward phase were better than the so-far best ones.

6. Consider the so-far best subset to be the current subset.

Backward Phase steps:

7. Initialize $\mathrm{o}=1$

8. Perform conditional exclusion. Find out the so-far best subset of size k-o.

9. Perform conditional increase of generalization step. The conditionally excluded features are returned.

10. None of the subsets tested in the backward phase were better than the so-far best ones go to forward phase.

\section{Pattern Matching Module}

\section{i. Pattern Matching using $k$-NN classifier}

In vein pattern matching a one - to - one match is done in between the registered pattern and the template already stored in the database to recognize the legitimate user which produces true of false result. $[3,4,5]$. This proposed multimodal palm vein recognition system uses the k-NN classifier with the Euclidian distance as the similarity measure. The $\mathrm{k}-\mathrm{NN}$ takes a test instance $\mathrm{x}$, finds it $\mathrm{k}$ nearest neighbors in the training data, and assigns $\mathrm{x}$ to the class occurring most often among those $\mathrm{k}$ neighbors. The algorithm can be described as follows:

- Out of $\mathrm{N}$ training vectors, identify the $k$ nearest neighbors based on Euclidian distance of $x$ irrespective of the class label. Choose a prime $k$.

- Identify the number of samples $k_{j}$ that belongs to class $w_{j}$ such that $\sum_{j} k_{j}=k$

- Assign $x$ to the class with the maximum number of $k_{j}$ samples.

Choosing the large $k$ has the gain of creating smooth resolution and also has higher computational complexity. This simply evaluates the mysterious data, for which it must have contact to the complete database. Therefore this approach is also called memory-based learning.

\section{ii. Pattern Matching using Adaptive Resonance Theory}

The k-NN classifier applied for recognition can compare the unknown palm vein pattern that can be the training pattern with the database. The database consists of the palm vein pattern of registered users. Hence this approach is lazy learning or memory based learning approach, which can be lead to increase the computational complexity and cost during the testing process. The proposed palm vein system can be done the pattern matching using ART1, which can learn and recognize the binary palm vein pattern. This ART1 matching module can be constructed by an attentional subsystem and an orienting subsystem. The attentional subsystem is responsible for competitive learning and enhancing the palm vein pattern by suppressing noise. The orienting subsystem can work as a novelty detector. Initially the bottom-up and top- down weights of ART1 can be set as

$$
\begin{aligned}
& B_{i j}=L(L-1+M) \\
& T_{i j}=1
\end{aligned}
$$

where $\mathrm{L}$ is the number of layers and $\mathrm{M}$ is the number of users. When the vigilance level is satisfied the bottom-up and top-down weights can be calculated by

$$
\begin{aligned}
& B_{i j}=2 \times T_{i j} \times x_{i} / l+s t_{i j} \times x_{i} \\
& T_{i j}=T_{i j}(\text { old }) \times x_{i}
\end{aligned}
$$

where $x i$ is the element in the input vector. The vigilance parameter is denoted by $\rho$, if the vigilance parameter value is large the it can be easy to find out the small difference between the input vectors with the patterns already stored in database. Other wise the more input patterns can be recognized into the same category and the recognition accuracy may low.

Algorithm steps:

1. Initialize the bottom up and top down weights, store the weights separately.

2. Set the vigilance level.

3. Get the optimized palm vein pattern as an input vector.

4. Perform a dot product between the bottom up weights and the input vectors, so that a neuron in the recognition layer fire arbitrarily.

5. Test the vigilance level with top down weights. Case A :

If the vigilance level is satisfied then the top down weights and bottom up weights have to be changed according to the below equations.

The bottom up weights can be changed as

$$
b_{i j}(n e w)=\frac{2 \times t_{i j} \times x_{i}}{1+s t_{i j} \times x_{i}}
$$

The top down weights can be changes as

$$
t_{i j}(n e w)=t_{i j}(\text { old }) \times x_{i}
$$

Case B:

- If the vigilance level is not satisfied the neuron will be inhibited totally from the competition and the next neuron will fire arbitrarily, and the vigilance level is tested once again. The process repeated until any neuron in the recognition layer satisfies the vigilance level. If none of the neuron is 
satisfied, a new location is allocated for the input vectors.

- After performing complete training on the ART, provide the new palm vein input vector for recognition, it can identify the palm vein pattern, which matches with the previously trained patterns. The legitimate user can be recognized accurately.

6. Store the newly changed bottom up and top down weights in their respective files.

\section{E. Binary Classifier}

The decision module is responsible to decide whether the enrollee is a legitimate user or an imposter. The decision should be based on the threshold value. If the matching score obtained from the k-NN classifier and ART are above the threshold value then the enrollee is a legitimate user, otherwise enrollee is an imposter. The high threshold value yields low acceptance rate and high rejection rate and high acceptance rate and low rejection rate are obtained by lower threshold value. Using trial and error basis select the optimal threshold value for the decision module to achieve the best palm vein recognition system. The proposed method constructs the decision module by the classifier based on binary decision rule using optimal threshold value, which hast to decide between two possible choices such as legitimate user or imposter.

TABLE 1. PERFORMANCE COMPARISON OF FILTERING TECHNIQUES

\begin{tabular}{|l|c|c|c|}
\hline \multicolumn{1}{|c|}{$\begin{array}{c}\text { Filter } \\
\text { Type }\end{array}$} & $\begin{array}{c}\text { Varia } \\
\text { nce } \boldsymbol{\sigma}\end{array}$ & $\begin{array}{c}\text { SNR } \\
\text { (dB) }\end{array}$ & \multicolumn{1}{|c|}{$\mathbf{A}_{\mathbf{z}}$} \\
\hline Median & 0.001 & 9.1358 & 0.90 \\
\hline Gaussian & 0.001 & 11.2999 & 0.92 \\
\hline $\begin{array}{l}\text { Osher - Rudin } \\
\text { (OR) shock filter }\end{array}$ & 0.004 & 13.5321 & 0.925 \\
\hline $\begin{array}{l}\text { Alverz Mazorra } \\
\text { (AM) shock filter }\end{array}$ & 0.006 & 15.7612 & 0.94 \\
\hline $\begin{array}{l}\text { GSZ Shock filter } \\
\text { (Real part) }\end{array}$ & 0.006 & 17.7041 & 0.99 \\
\hline
\end{tabular}

\section{F. Fusion At Decision Level}

Each k-NN classifier and ART provides a similarity score indicating the imminence of the input feature vector with the template feature vector. The recognition decision is based on its own matching score. The decision taken by each individual decision module is fused to find the final decision. The fusion on decision level is done by the Naive Bayes fusion approach. Each classifier makes an individual decision on the binary decision rule. The overall decision is simply the fusion of these classifiers using Naive Bayes fusion approach.The Naive Bayes (NB) fusion approach considers decisions of the individual classifiers are independent. Using $\mathrm{n}$ features $\mathrm{f}$ (individual classifier decisions) that picks a particular decision $\mathrm{d}$ of a set of Decisions $\mathrm{D}$, the NB decision rule is $\operatorname{classify}\left(f_{1} \ldots f_{n}\right) \arg \max _{c} p(D=d) \prod_{i=1}^{n} p\left(F_{i}=f_{i} / D=d\right)$

\section{RESUlts AND Discussion}

The proposed multimodal palm vein recognition is carried out on 250 samples of 50 users (5 samples of each user with different orientation). In this paper image denoising and enhancement is performed by GSZ shock filter. The real part of the GSZ shock filter provides the de-noised and enhanced image. The results of median filter and various shock filters are compared in terms of SNR (Signal to Noise Ratio) of de-noised image shown in Table 1. Fig 3 shows that GSZ shock filter improves the SNR better than the other filters. GSZ shock filter results in more visually appealing and adapts to discontinuities in images than other filters.

The performance analysis of palm vein recognition system with enhancement and without enhancement on palm vein images is done by the area under ROC (Receiver Operating Curve) shown in Table 1 and fig 4.

The hand shape feature is extracted from the palm vein image, and the fusion is based on a combination by convoluting the hand geometrical features and the palm texture to construct the multiple features set. The shape feature is extracted by the contour imaging. The palm texture characteristics are extracted by a Gabor filter. The hand shape feature, dominant points and the convolved multiple feature set are shown in fig 5 .

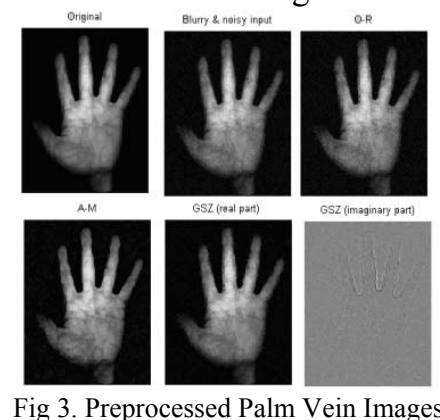

The palm vein features such as texture and shape with directional information are extracted for recognition. The direction information is extracted by performing skeletonization using the template proposed by S. Malki et al [9]. Skeleton of the palm vein is extracted applying thinning and pruning algorithms.Bifurcation and ending points are extracted from the skeleton of the palm vein. The binary images of the palm vein in eight directions are shown in fig 6 . The various extracted features are shown in the fig 7-9. Automatic subset selection is required to select the optimal feature set from the features obtained in eight directions.

Analysis of Classification Accuracy using Output of Various Filters

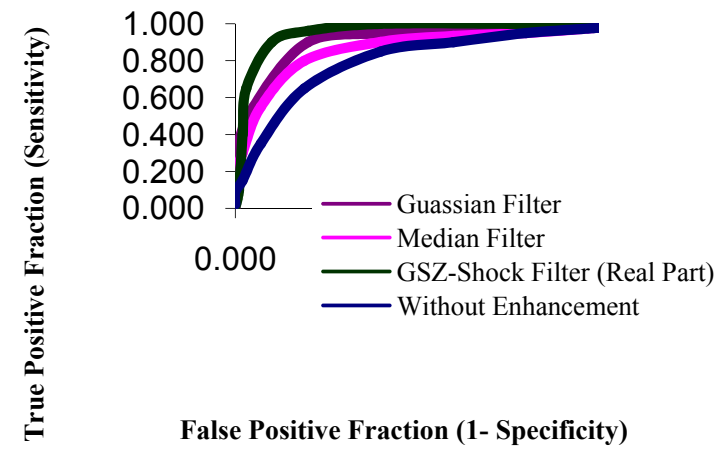

Fig 4. Analysis on Recognition Accuracy depends on various filtering techniques 


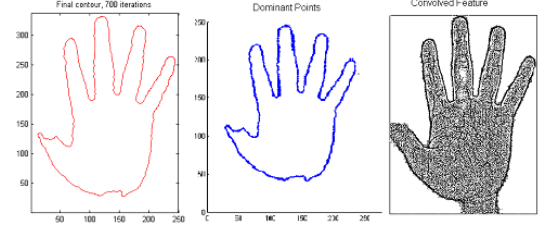

Fig 5. Shape, dominant points and convolved feature set

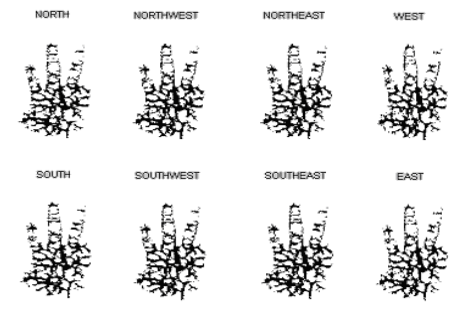

Fig 6. Binary image in eight directions

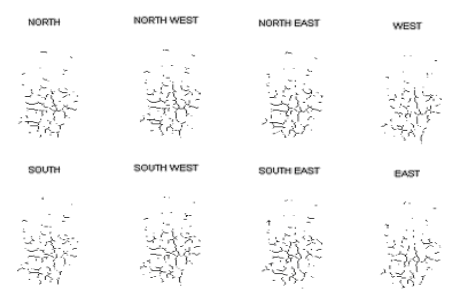

Fig 7. Skeleton of palm vein in eight directions

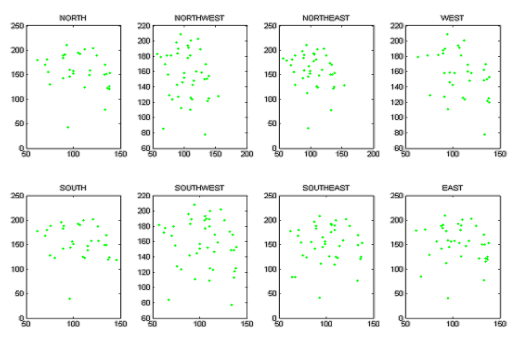

Fig 8. Bifurcation points in eight directions

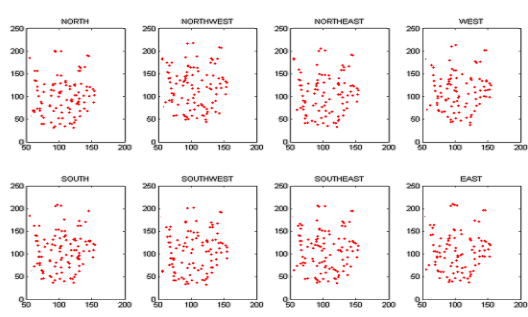

Fig 9. Ending points in eight directions

In this paper both the genetic algorithmic and Adaptive sequential floating forward search (ASFFS) approaches are considered to extract salient features from the multiple features set. The comparison of the genetic algorithms based feature selection methods (SGA \& CHC) and ASFFS feature selection method for palm vein pattern authentication is carried out. In this proposed system, the parameters set to the SGA (Simple Genetic Algorithm) are population size 60, maximum number of generation 7200 , cross over probability 0.75 , and mutation probability 0.01 . For CHC (Nontraditional Genetic Algorithm), population size 50, maximum number of generation 950 and large mutation rate 0.40 .

In ASFFS feature subset selection $r_{\text {max }}$ is a user specified absolute generalization limit set to 4 and the value of $b$ (neighborhood of final dimension) is set to 3 . Initially it is optimized by wrapper-based selection and LDA to convert it into lower dimensionality feature set. The multimodal palm vein recognition system reduces the computational complexity due to the less number of features and increases the acceptable accuracy. The optimal feature set, which is selected by ASFFS with LDA can improve the recognition accuracy and reduce the matching error percentage. The performance comparisons of these methods are done by ROC analysis. The overall ROC performance for various optimization techniques are shown in fig 10 as well as in Table 2. The k-NN classifier and Euclidean distance as the similarity measure do the pattern matching process. The large $\mathrm{k}$ value can obtain high recognition accuracy, which is selected by the trial and error basis. The $\mathrm{k}$ value used in this matching module is 90 .

The vigilance level used in this ART1matching module can affect the final recognition accuracy. The vigilance parameter is denoted by $\rho$, if the vigilance parameter value is large the it can be easy to find out he small difference between the input vectors with the patterns already stored in database. Other wise the more input patterns can be recognized into the same category and the recognition accuracy may low.

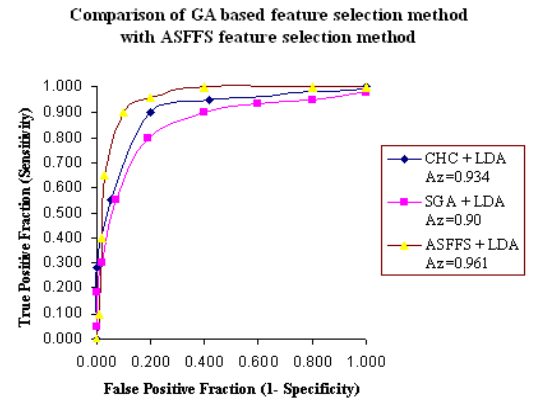

Fig 10. ROC analysis of optimization techniques

ROC Analysis on Palm Vein Recognition

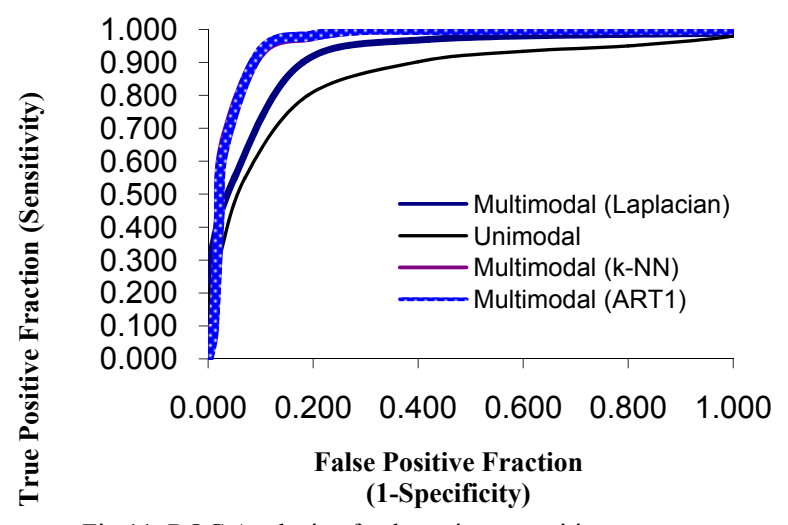

Fig 11. ROC Analysis of palm vein recognition systems 
TABLE 2: PERFORMANCE COMPARISON OF OPTIMIZATION TECHNIQUES

\begin{tabular}{|c|c|c|c|c|c|c|}
\hline \multirow[b]{2}{*}{$\begin{array}{c}\text { Feature Type / Optimization } \\
\text { Technique }\end{array}$} & \multicolumn{2}{|l|}{ SGA } & \multicolumn{2}{|l|}{$\mathrm{CHC}$} & \multicolumn{2}{|l|}{ ASFFS } \\
\hline & $\begin{array}{l}\text { Input } \\
\text { Features }\end{array}$ & $\begin{array}{c}\text { Optimal } \\
\text { Features } \\
\text { Selected }\end{array}$ & $\begin{array}{l}\text { Input } \\
\text { Features }\end{array}$ & $\begin{array}{l}\text { Optimal } \\
\text { Features } \\
\text { Selected }\end{array}$ & $\begin{array}{l}\text { Input } \\
\text { Features }\end{array}$ & $\begin{array}{l}\text { Optimal } \\
\text { Features } \\
\text { Selected }\end{array}$ \\
\hline Convolved Feature set & 322 & 47 & 322 & 29 & 322 & 34 \\
\hline Skeleton & 434 & 57 & 434 & 27 & 434 & 36 \\
\hline Bifurcation & 65 & 11 & 65 & 9 & 65 & 13 \\
\hline Ending Points & 78 & 19 & 78 & 13 & 78 & 16 \\
\hline
\end{tabular}

TABLE 3 : PERFORMANCE COMPARISON OF UNIMODAL AND MULTIMODAL PALM VEIN RECOGNITION

\begin{tabular}{|c|c|c|c|c|c|c|c|c|c|}
\hline Approach & NFA & NIVA & FAR (\%) & NFR & NEVA & FRR (\%) & EER & $\begin{array}{l}\text { MER } \\
\%\end{array}$ & RA \\
\hline Unimodal & 8 & $10^{5}$ & 8 e- 6 & 980 & $10^{5}$ & 0.0098 & $2.5 \%$ & 1.016 & $98 \%$ \\
\hline $\begin{array}{l}\text { Multimodal } \\
\text { (k-NN) }\end{array}$ & 3 & $10^{5}$ & $3 e-6$ & 400 & $10^{5}$ & 0.004 & $1.11 \%$ & 0.4 & $99.59 \%$ \\
\hline $\begin{array}{l}\text { Multimodal } \\
\text { (ART1) }\end{array}$ & 3 & $10^{5}$ & $3 e-6$ & 370 & $10^{5}$ & 0.0037 & $1.09 \%$ & 0.37 & $99.621 \%$ \\
\hline
\end{tabular}

TABLE 4: PERFORMANCE COMPARISON OF K-NN AND ART 1

\begin{tabular}{|c|c|c|c|c|c|c|}
\hline Approach & $\begin{array}{l}\text { Number } \\
\text { of Input } \\
\text { Neurons }\end{array}$ & $\begin{array}{c}\text { Number of } \\
\text { Output } \\
\text { Neurons }\end{array}$ & $\begin{array}{c}\text { Number of Hidden } \\
\text { Layers }\end{array}$ & Learning Rate & $\begin{array}{l}\text { Learning Time } \\
(\mu \mathrm{s})\end{array}$ & $\begin{array}{c}\text { Recognition } \\
\text { Accuracy }\end{array}$ \\
\hline k-NN & 25 & 1 & 1 & 0.85 & 0.53 & $99.59 \%$ \\
\hline ART1 & 25 & 1 & 0 & 0.95 & 0.34 & $99.621 \%$ \\
\hline
\end{tabular}

TABLE 5: EXECUTION TIME CALCULATION FOR LAPLACIANPALM VEIN RECOGNITION SYSTEM (5 SAMPLES OF EACH USER)

\begin{tabular}{|l|l|}
\hline Process Type & $\begin{array}{l}\text { Execution } \\
\text { Time (ms) }\end{array}$ \\
\hline Preprocessing & 40 \\
\hline Image registration & 100 \\
\hline Fusion (Imaging level) & 310 \\
\hline Feature Extraction & 18 \\
\hline Pattern Matching & $9(\mu \mathrm{s})$ \\
\hline Total Execution Time & $\mathbf{4 6 8} \mathbf{~ m s}$ and $9 \boldsymbol{\mu s}$ \\
\hline
\end{tabular}


Fusion at decision level is done by Naïve Bayes fusion approach with the decision values of four individual classifiers applied in the decision modules of each feature set such as convolved feature set, skeleton, bifurcation and ending points. By using trial and error basis the priority values are assigned to every feature. The higher priority value 3 is assigned to convolved feature set, the next priority value 2 is assigned to skeleton of the vein image and the lower priority value 1 is assigned to both bifurcation and ending points of the vein pattern. The performance comparison of unimodal and multimodal palm vein recognition systems are shown in Table 3 with respect to FAR, FRR, EER, MER and RA.

TABLE 6: EXECUTION TIME CALCULATION FOR MULTIMODAL PALM VEIN RECOGNITION SYSTEM CONSIDERING PATTERN MATCHING BY K-NN AND ART1 CLASSIFIERS (1

\begin{tabular}{|c|c|c|c|}
\hline \multicolumn{2}{|c|}{ Process Type } & \multicolumn{2}{|c|}{ Execution Time (ms) } \\
\hline \multicolumn{2}{|c|}{ Preprocessing } & \multicolumn{2}{|c|}{5} \\
\hline \multicolumn{2}{|c|}{ Feature Extraction } & \multicolumn{2}{|r|}{17.9154} \\
\hline \multicolumn{2}{|c|}{$\begin{array}{l}\text { Fusion at Extraction Level } \\
\text { (Convolution) }\end{array}$} & \multicolumn{2}{|r|}{6.534} \\
\hline \multicolumn{2}{|l|}{ Optimization } & \multicolumn{2}{|r|}{18.4219} \\
\hline $\begin{array}{c}\text { Pattern } \\
\text { Matching by } \\
\text { k-NN }\end{array}$ & 6.5242 & $\begin{array}{c}\text { Pattern } \\
\text { Matching by } \\
\text { ART1 }\end{array}$ & 2.5242 \\
\hline \multicolumn{3}{|l|}{ Decision } & 0.17388 \\
\hline \multicolumn{3}{|c|}{ Fusion at Decision Level } & 2.5454 \\
\hline $\begin{array}{l}\text { Total } \\
\text { (5 samples/ } \\
\text { user) }\end{array}$ & 285.5739 & $\begin{array}{l}\text { Total } \\
\text { (5 samples/ } \\
\text { user) }\end{array}$ & 265.5739 \\
\hline
\end{tabular}

Table 4 shows the performance comparison of k-NN and ART1 classifiers with respect to the key parameters learning rate, learning time and recognition accuracy. The performance comparisons of these methods are done by ROC analysis. The overall ROC performance of unimodal is $\mathrm{Az}=0.92$, Laplacianpalm is $\mathrm{Az}=0.945$, Multimodal recognition using $\mathrm{k}-\mathrm{NN}$ is $\mathrm{Az}=0.993$ and Multimodal recognition using ART1 is 0.995 which shown in Fig 11.

\section{Performance Comparison of Unimodal} and Multimodal

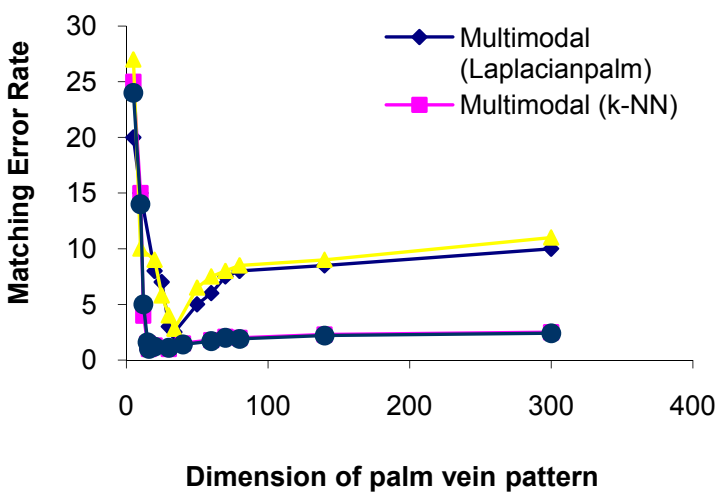

Fig 12. Comparison of Matching Error Rate

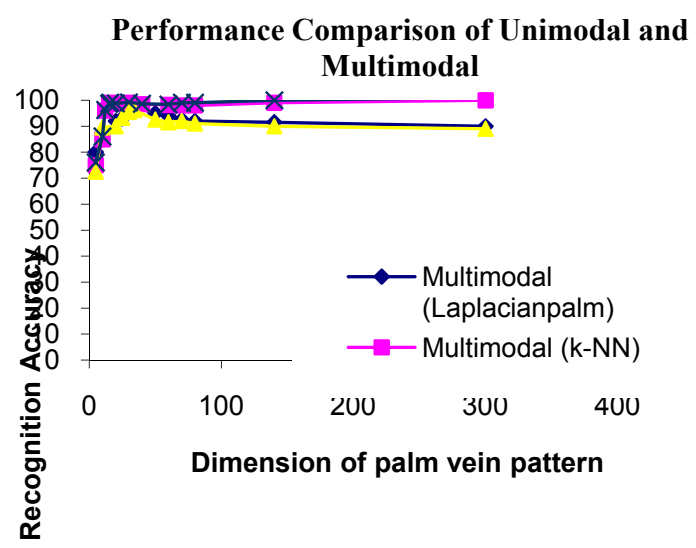

Fig 13. Comparison of Recognition Accuracy

The comparison of recognition accuracy (RA) and matching error rate (MER) for each optimal dimension in between unimodal and multimodal is shown in Fig $12 \& 13$. The execution times of these palm vein recognition systems are analyzed. The proposed multimodal palm vein recognition systems work faster than the Laplacianpalm multimodal palm vein recognition system, which is observed from Table 5 and Table 6.

\section{CONCLUSION}

The palm vein recognition system using multilevel fusion of multimodal features and neural network classifier has been developed. The shape and texture features have been extracted and multimodal features have been obtained at feature extraction level as well as matching score level. The Neural network classifier has been used to classify the vein patterns for making necessary decision. It is concluded from the analysis that the multimodal palm vein recognition system provides better performance compared unimodal features.

\section{REFERENCES}

[1] Fujitsu Computer Products of America, Inc., www.fcpa.fujitsu.com White Paper -"Palm Vein Authentication Technology ".2006.

[2] Mohamed Shahin, Ahmed Badawi, and Mohamed Kamel, "Biometric authentication using fast correlation of Near Infrared Hand Vein Patterns", International Journal of Biomedical Sciences, vol 2, No 3, 2007, ISSN 1306 - 1216.

[3] L.Wang and C.G Leedham, "A Thermal Hand Vein Pattern Verification System", In Lecture Notes in Computer Science, Springer, 2005.

[4] Jian-Gang Wang, Wei-Yun yau, Andy Suwandy, "Fusion of Palmprint and palm vein images for person recognition based on "laplacianpalm" feature", 1-4244-1180-7/07/ IEEE- 2007.

[5] Zhongli Wang, Baochang Zhang, Weiping Chen, Yongsheng Gao, "A performance Evaluation of Shape and Texture based methods for vein recognition", DOI 10.1109/CSIP.2008.106 - 978-0-76953119/08- IEEE - 2008.

[6] Chih-Lung Lin, Kuo-Chin Fan, "Biometric Verification Using Thermal Images of Palm-Dorsa Vein Patterns", IEEE Transactions on Circuits and Systems for Video Technology, Vol.14, No.2, Feb 2004.

[7] J.M.Cross, C.L.Smith, "Thermographic imaging of the subcutaneous vascular network of the back of the hand for biometric identification", 0-7803-2627-x/95-IEEE-1995

[8] Ding, Y., Zhuang, D. and Wang, K. " A study of Hand Vein Recognition Method", IEEE International Conference Mechatronics and Automation, pp. 2106-2110, 2005.

[9] Watanabe, M., Endoh, T., Shiohara, M, and Sasaki, S., "Palm Vein Authentication Technology and Its Applications", In Biometric Consortium Conference, USA, 2005. 
[10] Jian-Gang Wang, Wei-Yun yau, Andy Suwandy, "Feature Level Fusion of Palmprint and palm vein for person recognition based on a "Junction Point" Representation ", 978-1-4244-1764-3/08/IEEE2008.

[11] Chih-Lung Lin, Kuo-Chin Fan, "Biometric Verification Using Thermal Images of Palm-Dosra Vein Patterns", IEEE Transactions on Circuits and Systems for Video Technology, Vol.14, No.2, Feb 2004

[12] Weickert. J. "Coherence-enhancing Diffusion Filtering", International Journal of Computer Vision, Vol. 31(2/3), Pages 111$127,1999$.

[13] Guy Gilboa, Nir Sochen and Yehoshua Y. Zeevi, "Image Enhancement and Denoising by Complex Diffusion Processes", IEEE Transactions on Pattern Analysis and Machine Intelligence, Vol.26, No.8, 2004.

[14] Guy Gilboa, Nir Sochen and Yehoshua Y. Zeevi, "Regularized Shock Filters and Complex Diffusion", Springer - Verlag Nerling Heidelberg, ECCV 2002, LNCS 2350, pp 399-413, 2002.

[15] Suleyman Malki, Yu Fuqiang, Lambert Spaanenburg, "Vein Feature Extraction Using DT-CNNs", International Workshop on Cellular Neural Networks and Their Applications, IEEE 2006.

[16] Julien Doublet, Olivier Lepetit, Marinette Revenu, "Contact less Hand Recognition Using Shape and Texture Features”, ICSP2006 Proceedings.

[17] Somol. P, Pudil. P, Novovicova. J, Paclik. P, "Adaptive Floating Search Methods in Feature Selection", Pattern Recognition Letters 20 $1157-1163,1999$.

[18] Huiqing Chen, "Fingerprint image enhancement by diffusion processes", ICIP 2006, 1-4244-0481-9/06, IEEE 2006.

[19] C.Kamath, P.Miller, "Image Analysis for validation of simulations of Fluid Mix Problem", IEEE, International Conference on Image Processing. San Antonio, TX, USA, Sep 16, 2007

[20] H. P. Kramer and J. B. Bruckner, "Iterations of a non-linear transformation for enhancement of digital images", Pattern Recognition, 7:53-58, 1975.

[21] Ajay Kumar, David Zhang, "Personal Recognition using hand shape and texture", IEEE Transactions on Image Process, Vol. 8, 2454-61, 2006.

[22] Anil K.Jain, Arun Ross, Salil Prabhakar, "An Introduction to Biometric Recognition", IEEE Transactions on Circuits and Systems for Video Technology, Vol. 14, No. 1, 2004.

[23] Kwangcheol Shin, Ajith Abraham and Sang Yong Han, "Improving kNN Text Characterization by Removing outlines from Training set", Sprnger - Verlag Berlin Heideleberg, LNCS 3876, pp 563-566, 2006.

[24] Arun Ross, Anil Jain, "Information fusion in biometrics", Elsevier Science, DOI-10.10161/S0167-8655(03) 00079-5, 2003.

M.Deepamalar received MCA and M.Phil Degree from Bharathidasan University in 2004. At present she is pursuing Ph.D in Mother Teresa Women's University, Kodaikanal, India. And working as a Lecturer in Department of MCA, Muthayammal Engineering College, Rasipuram, India.

M.Madheswaran obtained his Ph.D degree in Electronics Engineering from the Institute of Technology, Banaras Hindu University, Varanasi, India, in 1999. At present he is a Principal of Muthayammal Engineering College, Rasipuram, India. He has authored over Seventy Five Research publications in international and national journals and conferences. Currently he is the chairman of IEEE India Electron Devices Society Chapter. He was awarded the Young Scientist Fellowship (YSF) by the State Council for Science and Technology, Tamilnadu in 1994 and Senior Research Fellowship (SRF) by the Council of Scientific and Industrial Research (CSIR), Government of India. He is a life member of IETE, ISTE and IE (India) and also a senior member of IEEE. 